\title{
Functional evaluation in high energy (Schatzker Type IV, V, VI) tibial plateau fractures treated by open reduction internal fixation
}

\author{
Nandkishor B. Goyal ${ }^{1}$, Ashish Vinayak Patil ${ }^{2 *}$, Swapnil Patil ${ }^{3}$ \\ ${ }^{\mathbf{1}}$ Associate Professor, ${ }^{2}$ Junior Resident, ${ }^{3}$ Senior Resident, Dept. of Orthopaedics, ACPM Medical College, Dhule, Maharashtra, India
}

*Corresponding Author: Ashish Vinayak Patil

Email: drashishpati188@gmail.com

\begin{abstract}
Introduction: Aim of the study is to find functional outcome of high energy (Schatzker type IV, V, VI) tibial plateau fracture after open reduction internal fixation and to study the complications following open reduction internal fixation.

Materials and Methods: This is a prospective study carried out at our institute on 50 patients having tibial plateau fractures Schatzker type IV, V, VI from November 2016 to December 2018. Results will be evaluated with respect to the frequency of side of injury, frequency of type of fracture, frequency of method of treatment, clinical results, radiological results and IKDC score.

Result: IKDC subjective knee evaluation score was within 37 to 60 with mean of 53.1 at 3 months follow-up. At 6 months the IKDC subjective knee evaluation was within 40 to 67 with mean of 58.6.

Conclusion: Open reduction internal fixation can restore the maximal joint stability and congruity. Although weight bearing is delayed till solid bone union is evident, patient should be encouraged to regularly carry out both passive and active range of movements of the affected knee joint for cartilage nourishment and preservation. At 6 months the IKDC subjective knee evaluation was within 40 to 67 with mean of 58.6 .
\end{abstract}

Keywords: Tibial plateau fracture, Schatzker, IKDC score.

\section{Introduction}

Knee joint is a complex joint as it is involved in varied functions like load bearing, walking, running, sitting etc. Meticulous clinical and radiological assessment is a must for proper management of the patient.

Fractures of the tibial plateau represent $1 \%$ of all fractures and approximately $8 \%$ of fractures occurring in the elderly. ${ }^{1,2}$ Tibial plateau fractures frequently result in poor functional outcome.

The goals ${ }^{3}$ in treating fractures of the tibial plateau are to restore the joint space, restore the correct mechanical alignment of the limb, achieve optimal healing of bone, tendon and ligaments and allow painless full range of motion of the knee.

The optimal treatment of tibial plateau fractures is controversial. They can be managed both by non-operative and operative methods.

For the high energy tibial plateau fractures (Schatzker type IV, V and VI) the ideal mode of treatment is always debatable. Conservative mode of management, open reduction and internal fixation, closed reduction and percutaneous fixation, hybrid external fixation have all been suggested. ${ }^{4}$

In this study we are analyzing prospectively the functional evaluation in high energy (Schatzker Type IV, V and VI) tibial plateau fractures treated by open reduction internal fixation and its complications.

\section{Aims and Objectives}

To study the functional outcome of high energy (Schatzker type IV, V, VI) tibial plateau fracture after open reduction internal fixation and to study the complications following open reduction internal fixation.

\section{Materials and Methods}

This is a prospective study carried out at our institute on 50 patients having tibial plateau fractures Schatzker type IV, V, VI from November 2016 to December 2018. After taking proper history and meticulous physical and neurovascular examination the patients were prepared for the surgery. Records were maintained with data sheet having name, age, sex, occupation, type of fracture etc. Open reduction and internal fixation was done. Pre-op and follow up X-rays were collected.

Results will be evaluated with respect to the frequency of side of injury, frequency of type of fracture, frequency of method of treatment, clinical results, radiological results and IKDC score.

\section{Inclusion Criteria}

1. The patients with diagnosed tibial plateau fracture (Schatzker Type IV, V, VI) treated with open reduction internal fixation.

2. Both males and females between the age of 18 years to 70 years.

\section{Exclusion Criteria}

1. Patients with head injury.

2. Medically unfit for surgery.

3. Patients with pathological fractures.

4. Injury to major arteries.

5. Patients with malignancy, on chemotherapy or radiotherapy.

6. Patients with metabolic diseases.

7. Immunocompromised patients.

Written informed consent were taken from all patients for publication. 


\section{Approach}

As per the plan, antero-lateral or posterior-medial approach was taken. Meniscus was preserved in all the cases and submeniscal arthrotomy was carried out to visualize the articular surface. Reduction done accordingly, checked \& fixed provisionally with $\mathrm{K}$-wires under the guidance of $\mathrm{C}$ arm. As per the plan Locking compression plate/ buttress plate was placed and $6.5 \mathrm{~mm}$ cancellous screws applied and cortical screws applied in the diaphysis. If required, percutaneous screws were applied on the medial side in lateral buttress plating to prevent the medial collapse of the articular surface.

Post operatively the limb is rested in a well padded above knee slab. Our routine post-operative protocol was carried out. Static Quadriceps exercise started from day 1 as tolerated by the patient. Once the pain and the tension in the operated site comes down they are started on gentle assisted motion. By the end of first week, patients are started on active range of motion. Sutures are removed on $12^{\text {th }}-14^{\text {th }}$ post-operative day. Patients are started on non-weight bearing crutch walking on discharge $\left(12^{\text {th }}-14^{\text {th }}\right.$ postoperative day).

\section{Follow up}

Patients were evaluated at the time of discharge, 1 month, 3 months and 6 months after surgery. Partial weight bearing was allowed only after 12 weeks. Functional evaluation was carried out with International Knee Documentation Committee (IKDC) Score at 3 months and 6 months after surgery.

\section{Result}

Maximum patients having tibial plateau fracture i.e. $46 \%$ were in 51 to 60 years age group while $40 \%$ were 41 to 50 years old. There were $4 \%$ patients either 21 to 30 years or 31 to 40 years old and $6 \%$ were 61 to 70 years of age.

Tibial plateau fracture was noted in $28 \%$ female and $72 \%$ male patients. There was male preponderance with female to male ratio of 1: 2.6. Occupation of Tibial plateau fracture was farming in $38 \%, 18 \%$ were labourers, $16 \%$ were housewives and $6 \%$ were Government servants or vendor while $2 \%$ were shopkeepers. While $14 \%$ patients were not involved in any work. Tibial plateau fracture in patients was due to fall in $26 \%$ while $74 \%$ had RTA. Tibial plateau fracture was on left limb in $64 \%$ while $36 \%$ had fracture on right limb. The high energy tibial plateau fracture was Schatzker Type IV in 56\%, type V in 36\% and $8 \%$ had type VI.

High energy tibial plateau fracture was managed with cancellous screw in 2\%, 18\% had Lateral buttress plate (LBP), $8 \%$ had LBP with bone graft (BG). Locking plate was done in $10 \%, 16 \%$ in locking plate with $\mathrm{BG}, 32 \%$ had Medial buttress plate (MBP) while $14 \%$ had MBP with BG. Bone grafting was needed in $38 \%$ patients of High energy tibial plateau fracture while $62 \%$ patients did not need bone graft.

Discharge from incision site indicating wound infection was present in $6 \%$ patients, none of the patients had neurovascular injury. After management, pain was noted in $32 \%$ patient at time of discharge, $22 \%$ had pain at 1 month which reduced to $2 \%$ at 3 months follow-up. Swelling was in $24 \%$ patients and $6 \%$ patients had superficial wound infection at time of discharge only. None of the patients had implant exposure at any follow-up. On Radiological examination all 50 patients had alignment at time of discharge, 1 month, 3 month follow-up examination. In two patients varus deformity was observed at $6^{\text {th }}$ month imaging studies. IKDC subjective Knee evaluation score was within 37 to 60 with mean of 53.1 at 3 months follow-up. At 6 months the IKDC subjective Knee evaluation was within 40 to 67 with mean of 58.6 .

All 50 patients had normal generalized laxity, alignment, patella position or patella subluxation or effusion at 3 months follow-up. Two patients had varus deformity at $6^{\text {th }}$ month imaging studies. All patients had Grade A effusion and ligament examination. On radiological examination, callus was seen.

Grade D evaluation grade was in $100 \%$ at 3 months. Final evaluation grade at 6 months follow-up was at Grade A in $30 \%$ patients, Grade B in $39 \%$ patients, Grade C in $22 \%$ and $10 \%$ had Grade D.

After 6 months follow-up examination, it was found that passive flexion ROM of affected side was within 90 to 135 degrees with mean of 121.7 degrees. The active flexion ROM at knee joint was within 90 to 130 degrees with 116.4 degrees mean after 6 months of high energy tibial plateau fracture management. There was normal passive and active flexion ROM on the normal side knee joint. There was statistically no significant ( $p>0.05)$ difference of pain, swelling in different Schatzker Type of Fracture at time of discharge. Superficial wound infection was in $50 \%$ patients in Schatzker Type VI fracture and $5.6 \%$ in type V while none with type IV fracture at time of discharge, which was statistically very highly significant $(\mathrm{p}<0.001)$. At 1 month follow-up examination there was statistically no significant ( $>0.05$ ) difference of pain, swelling, superficial infection, radiological examination of union status in different Schatzker type of fracture. In Schatzker Type IV fracture, at 6 months follow-up the evaluation was Grade A in $32.1 \%$, Grade B in $46.4 \%$ patients while $21.4 \%$ had Grade C. In Schatzker Type $\mathrm{V}$ fracture, final evaluation at 6 months follow-up was $33.3 \%$ in Grade A \& B, while $27.8 \%$ had Grade C. All patients with Type VI Schatzker Type fracture had Grade D evaluation at 6 months follow-up.

At 6 months follow-up mean IKDC subjective knee evaluation score in Type IV Schatzker fracture was 61.4 compared to 57.6 in Type V and 43.5 in type VI. There was statistically very highly significant $(\mathrm{p}<0.001)$ difference of higher mean IKDC subjective knee evaluation score in lesser Schatzker type of fracture. Mean passive ROM of index side was 124.8 degrees in Schatzker type IV fracture, 120.6 degrees in type $\mathrm{V}$ and 105.0 degrees in type VI. There was statistically significant $(\mathrm{p}<0.05)$ difference of lower passive ROM of index side in higher Schatzker type of tibial plateau fracture at 6 months follow-up. Mean Active ROM of index side was 120.0 degrees in Schatzker type IV 
fracture, 116 degrees in type $\mathrm{V}$ and 90 degrees in type VI. There was statistically very highly significant $(\mathrm{p}<0.001)$ difference of decreasing active ROM of index side in severe type of unstable fracture at 6 months follow-up. There was statistically no significant $(\mathrm{p}>0.05)$ difference of passive and active ROM on opposite side limbs at 6 months follow-up.

Case 1

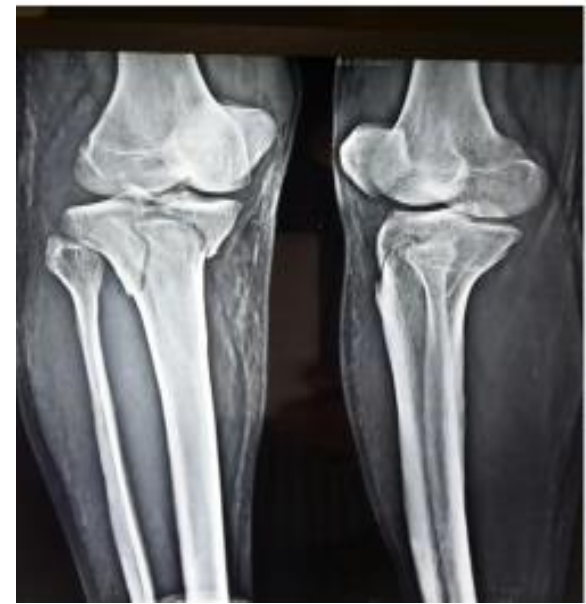

X-ray: Rt type V tibial plateau fracture 1 month Post-operative X- ray

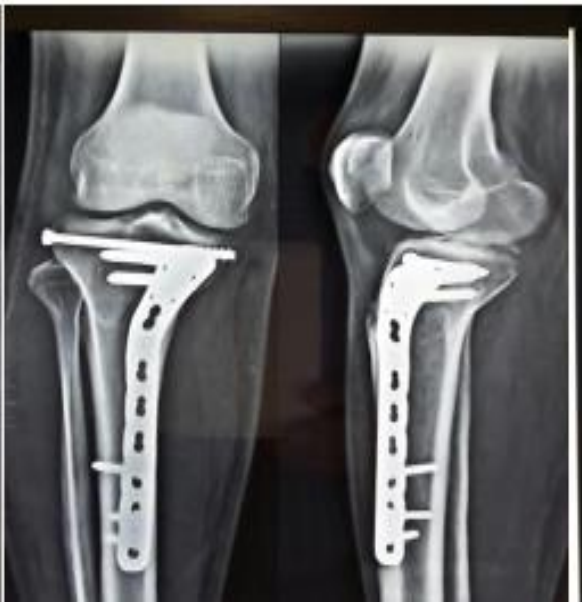

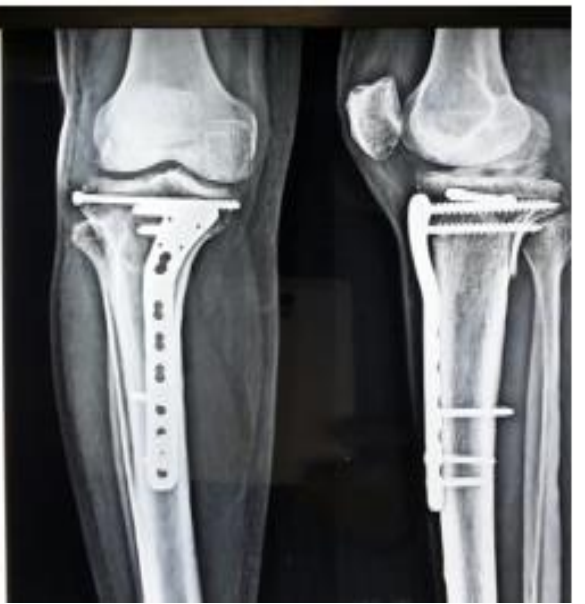

2 months Post-operative X-ray

\section{Intra Operative Photos}

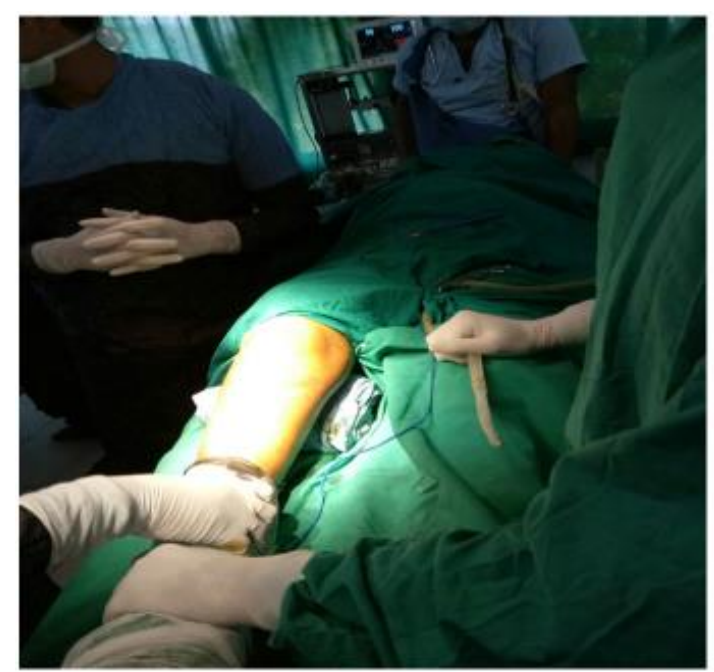

Patient position

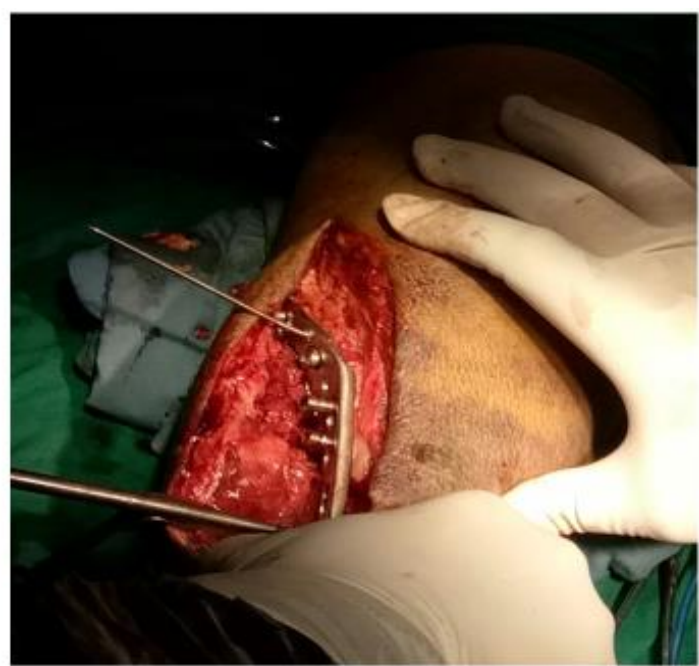

Intraoperative picture

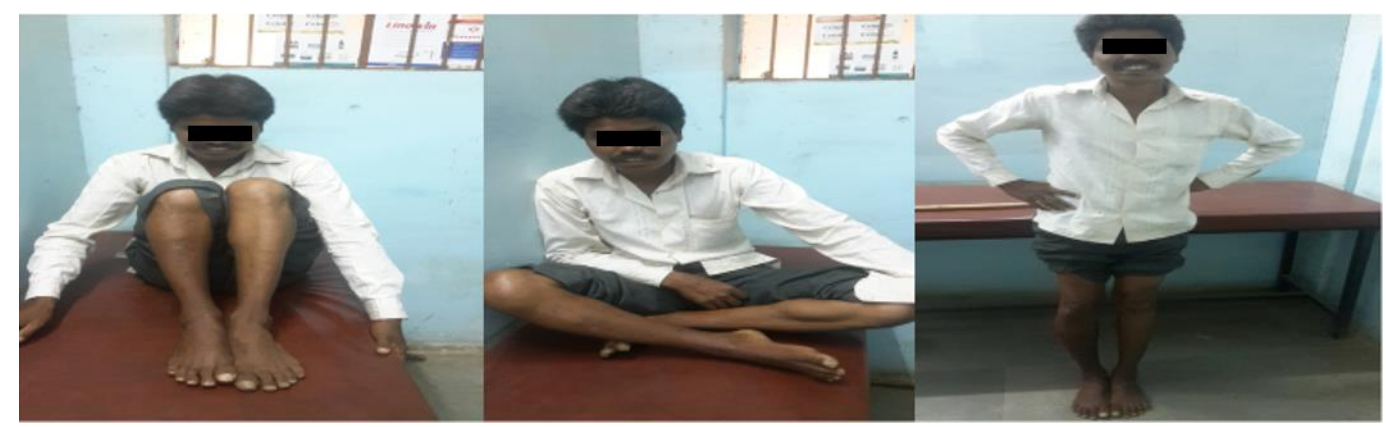

Functional outcome at 6 months follow up 


\section{Case 2}

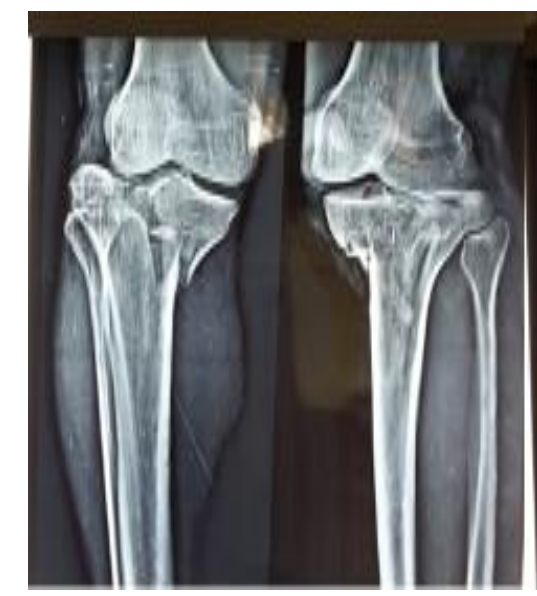

Type V Rt tibial fracture

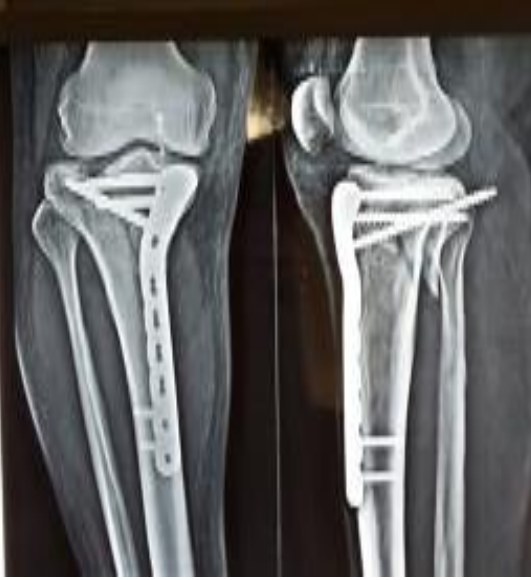

Immediate Post-operative X-rays

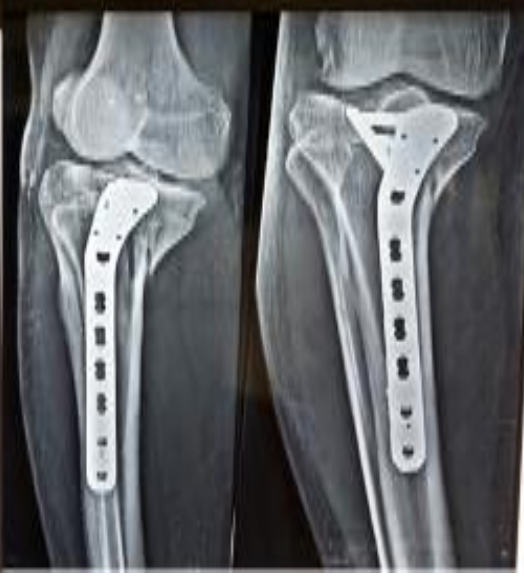

3 months follow up X-ray

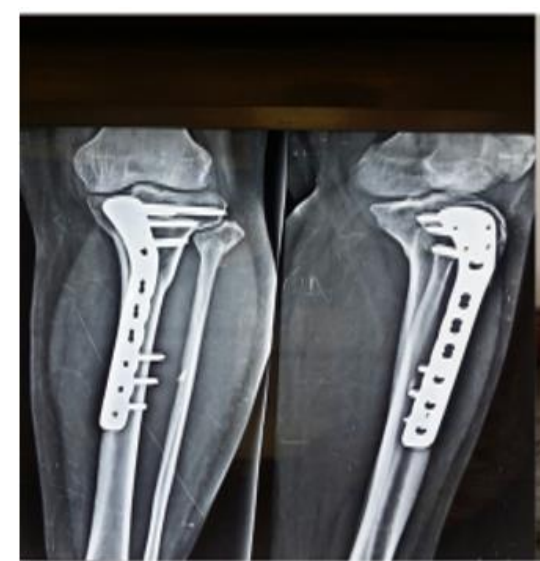

6 months follow up X-ray

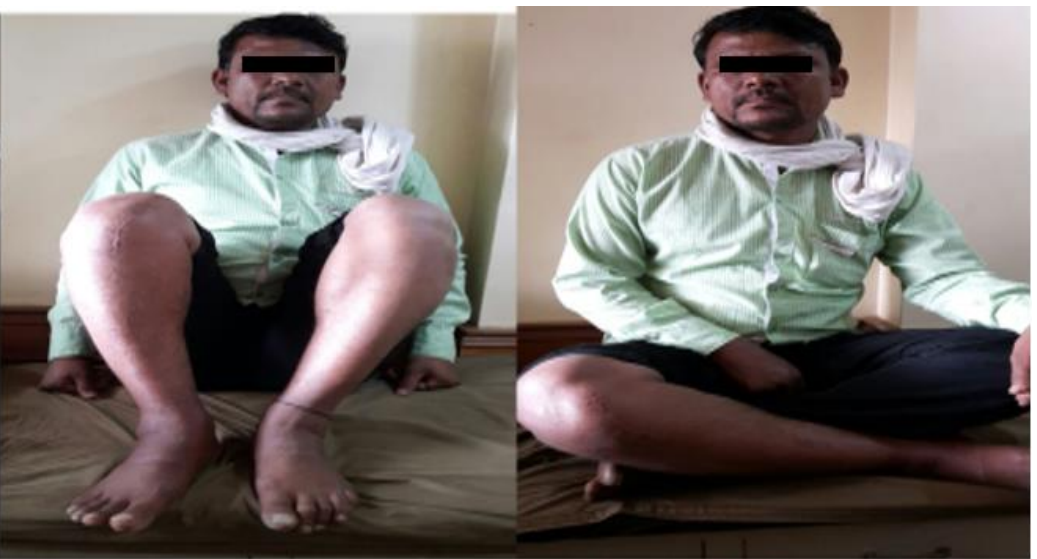

Functional outcome - 6 months follow up

Case 3

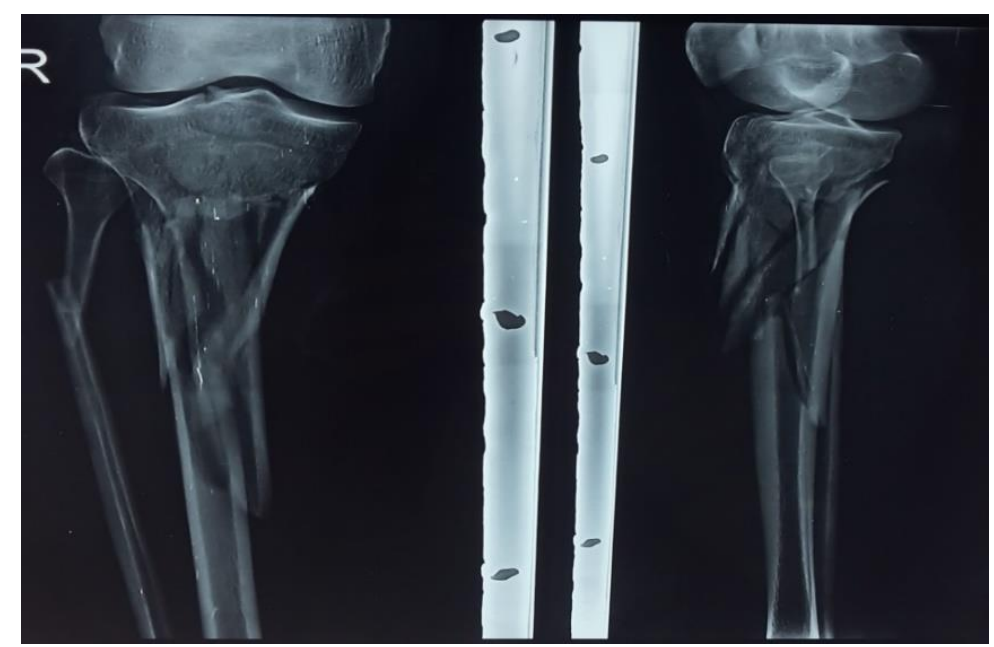

Preoperative X-ray 


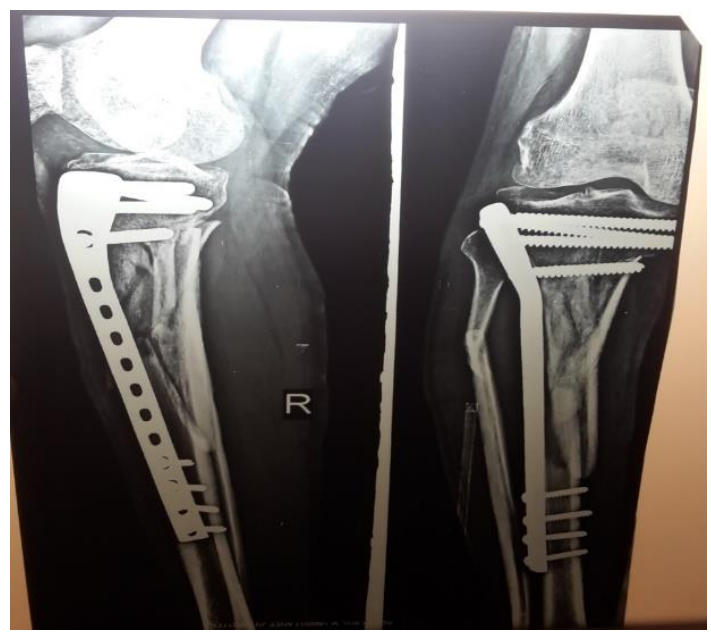

Postoperative X-ray

\section{Case 4}

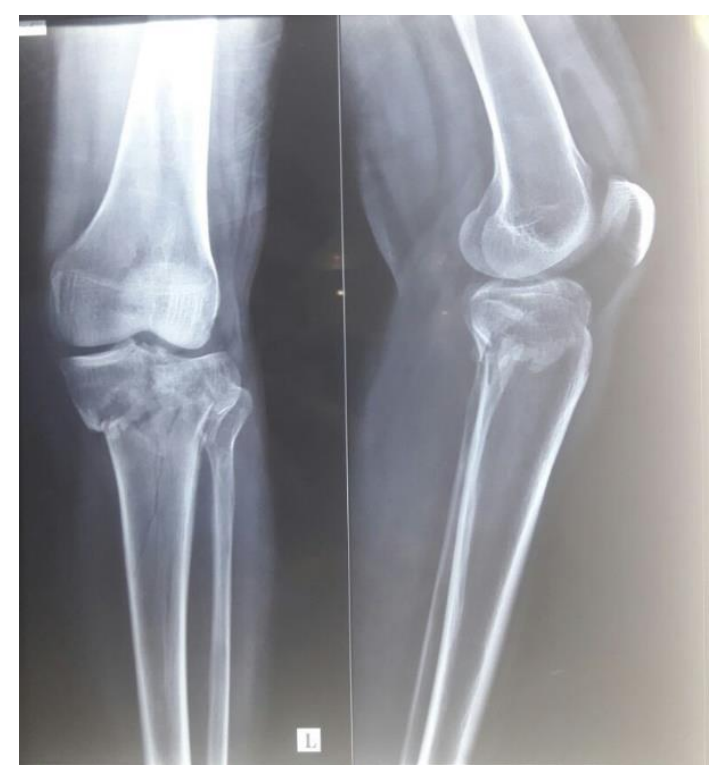

\section{Preoperative X-ray}

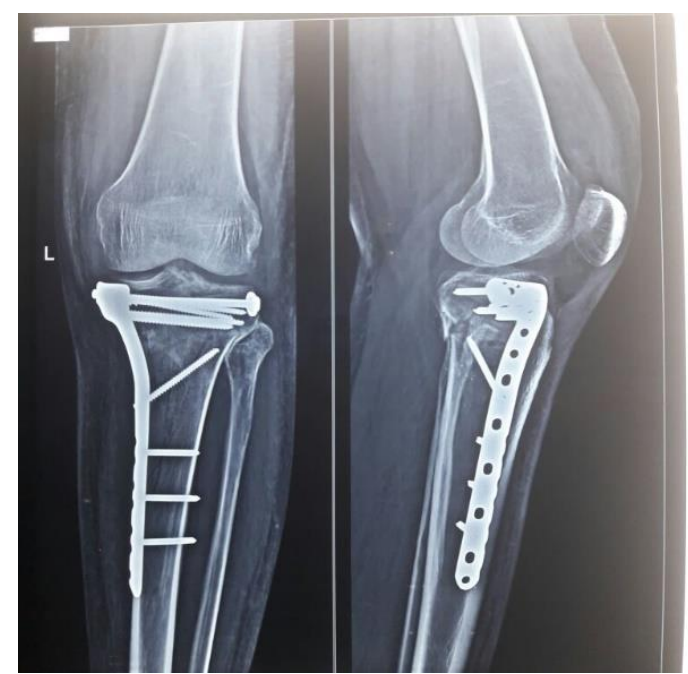

Postoperative X-ray

\section{Case 5}

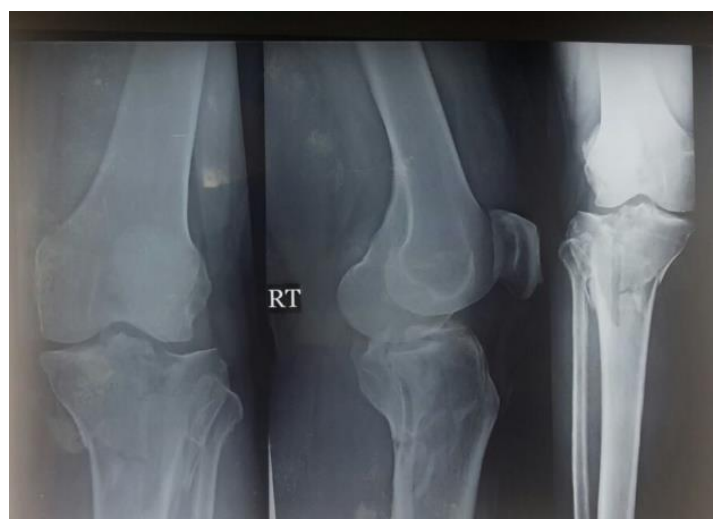

Preoperative X-ray

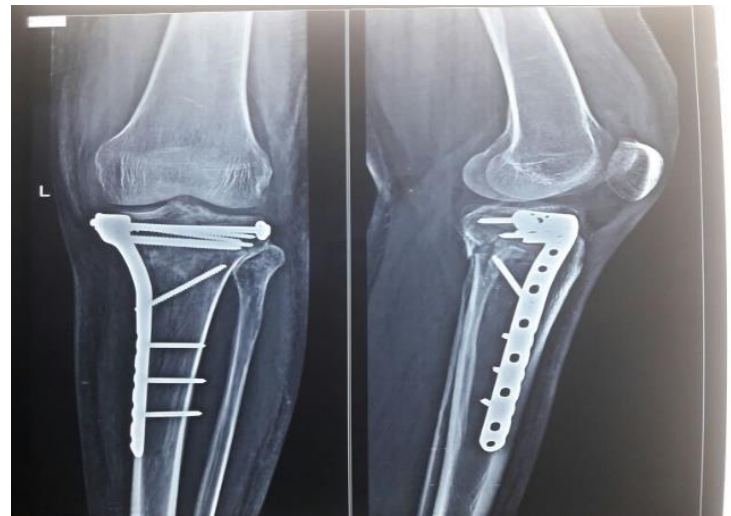

Postoperative X-ray

\section{Discussion}

This is a prospective study carried out at our institute on 50 patients having high energy tibial plateau fractures Schatzker type IV, V, VI from November 2016 to December 2018. Tibial plateau fractures are complex intraarticular fractures accounting for about $1 \%$ of all fractures. ${ }^{5}$ The main goal in treatment of tibial plateau fractures is to obtain painless, stable knee joint with a functional range of motion. ${ }^{6}$ Anatomical restoration of tibial joint surface is necessary to achieve treatment goal..$^{7,8}$ Stable internal plate fixation without damaging the soft-tissue envelope is very difficult to achieve. ${ }^{9}$

\section{Age and Sex Distribution}

In this study, maximum patients having tibial plateau fracture i.e. $46 \%$ were in 51 to 60 years age group while $40 \%$ were 41 to 50 years old. There was male preponderance with male to female ratio of 2.6:1. Occupation of patients was farming in $38 \%$ and $18 \%$ were labourers.

\section{History and Physical Examination}

One can know about open or closed fracture by inspection of soft tissue envelope. A high energy injury which precludes an immediate extensile exposure is indicated by clinical signs such as blisters, superficial abrasions, deep contusions and degloving injuries. ${ }^{10}$ The majority of tibial plateau fractures reported in the recent literature have 
resulted from high speed motor vehicle accidents and fall from height. ${ }^{11-13}$

In this study, tibial plateau fractures occurred due to fall in $26 \%$ while $74 \%$ had road traffic accident. Tibial plateau fracture was on left limb in $64 \%$ while $36 \%$ had fracture on right limb.

\section{Classification}

The Schatzker classification and AO/OTA Fracture and Dislocation Classification, are commonly used. A CTbased, three-column classification combined with injury mechanism provides guidance in the treatment of complex tibial plateau fractures. ${ }^{14}$ In this study, we used Schatzker classification.

\section{Timing of Surgery}

Skin wrinkling indicating regression of oedema is a good clinical indicator that it is safe to perform open reduction and internal fixation (ORIF). ${ }^{10}$ In our study, we waited on an average 5 to 7 days for the swelling to subside. Comorbid conditions like hypertension, diabetes, bronchial asthma etc. if any were managed for optimization of the patient.

\section{Aftercare}

Isometric quadriceps exercises are started as early as possible after surgery. ${ }^{10}$ Fractures caused by extremely high energy: these patients need to adhere to toe-touch weight bearing for 10-12 weeks but have active flexion throughout the last 10 weeks of this period..$^{10}$ Wound complications are the major early problems related with the management of high-energy tibial plateau fractures. ${ }^{10}$ In this study, after care involved monitoring for compartment syndrome and neurovascular status especially in the first 48 hours after surgery. Discharge from incision site was present in $6 \%$ patients, none of the patients had neurovascular injury in initial 48 hours in our study.

Assessment of patients at the time of discharge revealed $32 \%$ patients had pain, 24\% had swelling and 6\% had superficial infection. The superficial infection healed with the help of oral and topical antibiotics, debridement and regular dressing. Partial weight bearing was started at 3 months in this study.

\section{Joint Laxity}

Jansen et al. reported that in tibial plateau fractures, instability was observed in pivot shift test. ${ }^{7}$ Rademakers et al. followed patients for 14 years and observed $14 \%$ patients had anteroposterior laxity. ${ }^{15}$ In this study we found that there was no difference in laxity between the index and normal knees. Studies have reported increased meniscal and ligamentous injury with higher Schatzker types of fractures. ${ }^{16}$ Contrary to this, some studies reported no correlation between meniscal, ligamentous injury with fracture type. ${ }^{17,18}$ In our study, all 50 patients had normal generalized laxity, patella position or patella subluxation or no effusion at 3 months and 6 months follow-up. All patients had Grade A effusion and ligament examination. There was varus deformity observed in two patients at $6^{\text {th }}$ month follow-up imaging studies.

\section{Range of Motion}

Erdil M, Yildiz F, Kuyucu E, Sayar S, Polat G, Ceylan H., Kocyigit $\mathrm{F}$ reported in their study increase of fracture type was correlated with significant decrease in flexion angles ( $p$ $=0.021) \cdot{ }^{19}$ In tibial plateau fractures functional recovery increases with increased range of movement. ${ }^{8,20}$ Higher range of movement resulted in higher IKDC scores in our study. In our study, the final knee evaluation report is Grade $\mathrm{D}$ in $100 \%$ patients at 3 months follow-up. Final evaluation grade at 6 months follow-up was at Grade A in 30\% patients, Grade B in $39 \%$ patients, Grade C in $22 \%$ and $10 \%$ had Grade D.

After 6 months follow-up examination it was found that passive flexion ROM of affected side was within 90 to 135 degrees with mean of 121.7 degrees. The active flexion ROM at knee joint was within 90 to 130 degrees with 116.4 degrees mean after 6 months of high energy tibial plateau fracture management. There was normal passive and active flexion ROM on the normal side knee joint.

Erdil M, Yildiz F, Kuyucu E, Sayar S, Polat G, Ceylan $\mathrm{H}$., Kocyigit $\mathrm{F}$ reported in their study that knee flexion was statistically significantly decreased on the affected side compared to the healthy side $\left(100.16 \pm 16.14^{\circ}\right.$ versus $110.16 \pm 13.11, \mathrm{p}<0.001) .{ }^{19}$ Mean IKDC was $67.83 \pm$ 19.98. IKDC ( $p=0.001)$ tend to increase as the amount of knee flexion increased. ${ }^{19}$

In our study IKDC subjective Knee evaluation score was within 37 to 60 with mean of 53.1 at 3 months followup. At 6 months the IKDC subjective Knee evaluation was within 40 to 67 with mean of 58.6.

\section{Post-operative Imaging}

We were able to achieve excellent reduction and there was no malalignment noticed in immediate post-operative, $1^{\text {st }}$ month and $3^{\text {rd }}$ month follow-up imaging studies in any of the patients. There was bone union in all patients at $3^{\text {rd }}$ month. There was varus collapse observed in two patients at 6 month follow-up imaging studies.

\section{Comparison of different tibial plateau types at the time of discharge}

There was statistically no significant difference of pain, swelling in different Schatzker type of fracture at time of discharge. Superficial wound infection was in $50 \%$ patients in Schatzker type VI fracture and $5.6 \%$ in type V while none with type IV fracture at time of discharge, which was statistically very highly significant.

Comparison of different tibial plateau types at $1^{\text {st }}$ month follow up:

At 1 month follow-up examination there was statistically no significant difference of pain, swelling, radiological examination of union status in different Schatzker type of fracture.

\section{Comparison of different tibial plateau types at $6^{\text {th }}$ month follow up}

There was statistically very highly significant difference of poorer grades of passive and active motion deficit with 
higher Schatzker type of fracture at 6 months follow-up. There was statistically very highly significant difference of poor final grades of evaluation as per IKDC subjective knee evaluation with higher Schatzker type of fracture at 6 months follow-up. At 6 months follow up, there was statistically very highly significant difference of higher mean IKDC subjective knee evaluation score in lesser Schatzker type of tibial plateau fracture. There was statistically significant difference of lower passive and active ROM of index side in higher Schatzker type of fracture at 6 months follow-up. Our results are consistent with the literature.

There was varus collapse observed in two patients at $6^{\text {th }}$ month follow-up imaging studies. These two patients had Schatzker type V and type VI tibial plateau fractures and were treated by lateral buttress plating and lateral buttress plating with bone grafting respectively. Excellent anatomical reduction was achieved at time of surgery. There was no malalignment at 3 months follow-up imaging studies. Then there varus deformity observed at $6^{\text {th }}$ month follow-up imaging studies. But the patient had good range of movement. This is comparable with the series of Eggli et al., ${ }^{21}$ who had one case of varus collapse and one case of valgus collapse in their series of 14 patients with an average of 25 months follow up. In the series of Barei et al. ${ }^{22}$, of 31 patients studied, 28 patients had satisfactory coronal alignment, but two patients developed varus malalignment and one developed valgus malalignment.

\section{Long term follow up}

An essential goal of surgery is to provide stable fracture fixation that allows early joint mobilization ${ }^{10}$. Failure to achieve this will often result in poorer outcomes than nonoperative treatment. ${ }^{10}$

Non-union and deep infection are more frequent after ORIF of high-energy bicondylar tibial plateau fractures treated with dual plating through two incisions. ${ }^{22}$ The importance of restoring bone alignment, ligamentous stability, and meniscal preservation is emphasized in studies reporting outcomes. ${ }^{10}$ Removal of menisci, articular incongruity, or varus malreduction increased the percentage of degenerative arthritis in patients with more than 7 years follow-up ${ }^{23}$. Knee stability is believed to be the most important factor for long-term patient outcome. ${ }^{23}$ Stable joint allowing early range of motion is necessary for cartilage nourishment and preservation. ${ }^{24}$

In the experience of Schatzker et al, ${ }^{26} 78 \%$ of tibial plateau fractures treated surgically had satisfactory results after 2.3 years. Lachiewicz and Funcik $^{27}$ examined the operative treatment of patients with 43 tibial plateau fractures and found $81 \%$ excellent results after 2.7 years.

\section{Conclusion}

Open reduction internal fixation can restore the maximal joint stability and congruity. Although weight bearing is delayed till solid bone union is evident, patient should be encouraged to regularly carry out both passive and active range of movements of the affected knee joint for cartilage nourishment and preservation. At 6 months the IKDC subjective knee evaluation was within 40 to 67 with mean of 58.6 .

\section{Conflict of Interest: None.}

\section{References}

1. Jacofsky DJ, Haidukewych GJ. Tibial Plateau Fractures: Insall Scott Surgery of the knee; ch - 66;1133-1146;4th edition;2006

2. Wiss DA, Watson JT, Johnson EE, Fractures of the knee. In: Rockwood CA, Green DP, Bucholz RW, et al: Fractures in adults: ed 4 Philadelphia: Lipincott - Raven 1996, p.1999.

3. William J. Mills MD, and Sean E. Norle, MD: ORIF of high energy tibial plateau fractures. OCNA. 2002:33(1):177.

4. Koval KJ, Helfel DL. Tibial Plateau fractures-Evaluation and treatment. J Am Acad Orthop Surg 1995;3(2):86-94.

5. Ariffin HM, Mahdi NM, Rhani SA, Baharudin A, Shukur MH: Modified hybrid fixator for high-energy Schatzker V and VI tibial plateau fractures. Strategies Trauma Limb Reconstr 2011;6:21-6.

6. WATSON J. T. High energy fractures of tibial plateau. Orthop Clin North Am 1994;25:723-52.

7. Jansen H., Frey S. P., Doht S., Fehske K., Meffert R.H. Medium-term results after complex intra-articular fractures of the tibial plateau. J Orthop Sci 2013;18:569-77.

8. STEVENS D.G., BEHARRY R., MCKEE M.D., WADDELL J. P., SCHEMITSCH E. H. The long-term functional outcome of operatively treated tibial plateau fractures. J Orthop Trauma, 2001;15:312-20.

9. Weiner LS, Kelley M, Yang E, Steuer J, Watnick N, Evans M, Bergman M. The use of combination internal fixation and hybrid external fixation in severe proximal tibia fractures. $J$ Orthop Trauma 1995;9:244-50.

10. Cong-Feng L. Tibia, proximal. In: Buckley RE, Moran CG, Apivatthakakul T (Eds). AO principles of fracture management. $3^{\text {rd }}$ ed. New York: Theime: 2017. p877-97.

11. Jacofsky D J, Haidukewych G J. Tibial Plateau Fractures: Insall Scott Surgery of the knee; ch - 66;1133-1146;4th edition;2006

12. Mills W J, and Norle S E : ORIF of high energy tibial plateau fractures. OCNA 2002;33(1):177.

13. Kenneth A, Egol \& Kenneth J. Koval. Fractures of the proximal tibia: 1999- 2029; 6th edition 2006.

14. Zhu Y, Yang G, Luo CF. Computed tomography-based ThreeColumn Classifcation in tibial plateau fractures: introduction of its utility and assessment of its reproducibility. J Trauma Acute Care Surg. 2012;73(3):731-7.

15. Rademakers M. V., Kerkhoffs G. M., Sierevelt I. N., Raaymakers E. L., Marti R.K. Operative treatment of 109 tibial plateau fractures: five- to 27 -year follow-up results. J Orthop Trauma 2007;21:5-10.

16. STANNARD J. P., LOPEZ R., VOLGAS D. Soft tissue injury of the knee after tibial plateau fractures. J Knee Surg 2010;23:187-92.

17. BENNETT, W. F., BROWNER, B.: Tibial plateau fractures: a study of associated soft tissue injuries. J Orthop Trauma 1994;8:183-8.

18. ZAKRZEWSKI P., ORŁOWSKI J. Meniscuses and ligaments injuries in tibial plateau fractures in comparative evaluation of clinical, intraoperative and MR examination. Chir Narzadow Ruchu Ortop Pol, 2005;70:109-13.

19. ERDIL M, YILDIZ F, KUYUCU E, SAYAR S, POLAT G, CEYLAN H., KOÇYIĞIT F. The Effect of Sagittal Plane Deformities after Tibial Plateau Fractures to Functions and Instability of Knee Joint. Acta Chir Orthop Traum čech 2016;83(1):43-6. 
20. CHOO K. J., MORSHED S. Postoperative complications after repair of tibial plateau fractures. J Knee Surg 2014;27:11-9.

21. Eggli et al. Unstable bicondylar tibial plateau fractures: A clinical investigation. J Orthop Trauma 2008;22:673-9.

22. Barei. Functional outcomes of severe bicondylar tibial plateau fractures treated with dual incisions and medial and lateral plates. J Bone Joint Surg [Am] 2006;88A:1713-21.

23. Ruffolo MR, Gettys FK, Montijo HE. Complications of highenergy bicondylar tibial plateau fractures treated with dual plating through 2 incisions. J Orthop Trauma 2015;29(2):8590.

24. Giannoudis PV, Tzioupis C, Papathanassopoulos A. Articular step-off and risk of post-traumatic osteoarthritis. Evidence today. Inj 2010;41(10):986-95.

25. Lansinger O, Bergman B, Korner L et al (1986) Tibial condylar fractures. A twenty - year follow - up. J Bone Joint Surg Am 1986;68:13- 9.

26. Schatzker J, McBroom R, Bruce D. The tibial plateau fracture. The Toronto Experience 1968-1975. Clin Orthop 1979;138:94104.

27. Lachiewicz PF, Funcik T. Factors influencing the results of open reduction and internal fixation of tibial plateau fractures: Clin Orthop 1990;259:210-5.

How to cite this article: Goyal NB, Patil AV, Patil S. Functional evaluation in high energy (Schatzker Type IV, V, VI) tibial plateau fractures treated by open reduction internal fixation. Indian J Orthop Surg 2019;5(2):128-35. 